\title{
Numerical and Physical Modelling of Bubbly Flow Phenomena
}

\section{Final Report for the Department of Energy Grant DE-FG02-89FR14043}

Period of Performance: 07/01/1989 - 01/01/1999

\section{P.I.: A. Prosperetti}

Department of Mechanical Engineering The Johns Hopkins University, Baltimore MD 21218

prosperetti@jhu.edu

\section{Contents}

Summary of Results $\quad 2$

$\begin{array}{ll}\text { Publications Resulting from This Grant } & \mathbf{7}\end{array}$

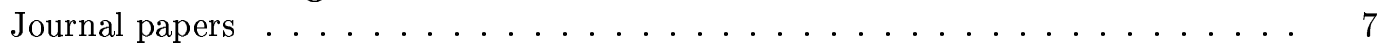

Papers in volumes $\ldots \ldots \ldots \ldots \ldots \ldots \ldots \ldots$

$\begin{array}{lr}\text { Personnel } & 9\end{array}$

$\begin{array}{lr}\text { Patent Report } & 9\end{array}$

$\begin{array}{lr}\text { Equipment Inventory } & 9\end{array}$

$\begin{array}{ll}\text { References } & 10\end{array}$ 


\section{Summary of Results}

The focus of this work has been the use of direct numerical simulations of spheres suspended in Stokes or potential flows to systematically develop closure relations for averaged equations describing the flow of fluids with suspended particles. Our efforts during the initial part of the project served to prepare the stage for the results in the last several years, which are the most interesting ones found in the course of this work. Accordingly, in the description that follows, we will focus on these latter results referring the reader to the publications listed below for the earlier work.

1. A distinctive feature of our efforts has been the study of spatially non-uniform systems, for which we had to develop a completely new approach. The reason we believe the study of such systems is of crucial importance for the development of averaged equations is a result that may be termed the Stability-Hyperbolicity Theorem (Prosperetti \& Jones 1985; Prosperetti \& Satrape 1990). This is a general result applicable to a very broad class of averaged-equations models that essentially encompasses all the models in current use. It states that, for all the models in this broad class, lack of hyperbolicity implies linear instability (but not vice versa). The result was originally derived for models containing only first-order spatial or temporal derivatives. For models with derivatives of higher order, it still implies that long wavelengths will be unstable unless the model, truncated to its first-order differential form, is hyperbolic (Jones \& Prosperetti 1987).

This result illuminates the crucial importance of differential terms in the averaged equations which has been recognized by others (see e.g. Batchelor 1988). While some such terms can be found by considering spatially uniform situations (e.g. the Newtonian part of the suspension stress with the attendant effective viscosity - see e.g. Batchelor 1970; Mo \& Sangani 1994, and also Sangani \& Didwania 1993; Bulthuis et al. 1995) not all can be obtained in this way. A typical simple example is the spatial part of the acceleration in the added-mass interaction. It is therefore necessary to consider explicitly spatially non-uniform systems, the study of which is much less developed than that of uniform ones. Specifically, while standard techniques exist for the numerical approximation of infinite uniform suspensions or composites (see below), no such technique existed for the non-uniform case prior to our work.

2. Before continuing, it is useful to demonstrate in a simple example that spatially non-uniform systems can differ not only quantitatively, but qualitatively from uniform ones, which greatly complicates the task of finding closure relations for them.

Consider a heat conduction problem in a composite consisting of 3 blocks. The gray block has infinite thermal conductivity while that of the white blocks is equal and finite (Fig. 1). The system can therefore exists in 3 states. It is required to determine the ensemble-average temperature at the midpoint. In the state of the system in which the gray block is in the middle, the temperature at the midpoint is 0.5 . If the gray block is on the left or the right, the temperature at the midpoint will be smaller or greater than 0.5 by the same amount $\delta$. Only the configuration with the gray block in the middle contributes to the ensemble-mean temperature in the gray material, which is therefore 0.5 . Both of the other two configurations contribute, however, to the ensemble-mean temperature in the other material at the midpoint. If they are equally probable, the phase average temperature for the other material will clearly be 0.5 as well. This result is in keeping with the well-known fact that, in a uniform composite, the ensemble-mean temperatures in the two phases are equal, which enables the mean heat flux to be expressed in terms of the gradient of a single mean temperature. If, however, the probability distribution of the finite-conductivity material in the ensemble is not uniform so that, for example, the configuration with the gray block on the left occurs with greater probability than the other one, the mean temperature will be less than 0.5 and, conversely, it will be greater than 0.5 if the gray block is more frequently on the right. In this case there will be a difference between the two mean phase temperatures and, for the more general case of spheres embedded in a matrix, we have shown that now the average heat flux vector will contain two contributions, one of which is proportional to the gradient of the phase-mean temperature difference (Marchioro \& Prosperetti 1998). This effect would have been impossible to anticipate solely on the basis of experience with uniform composites and, indeed, earlier attempts to deal with non-uniform systems 

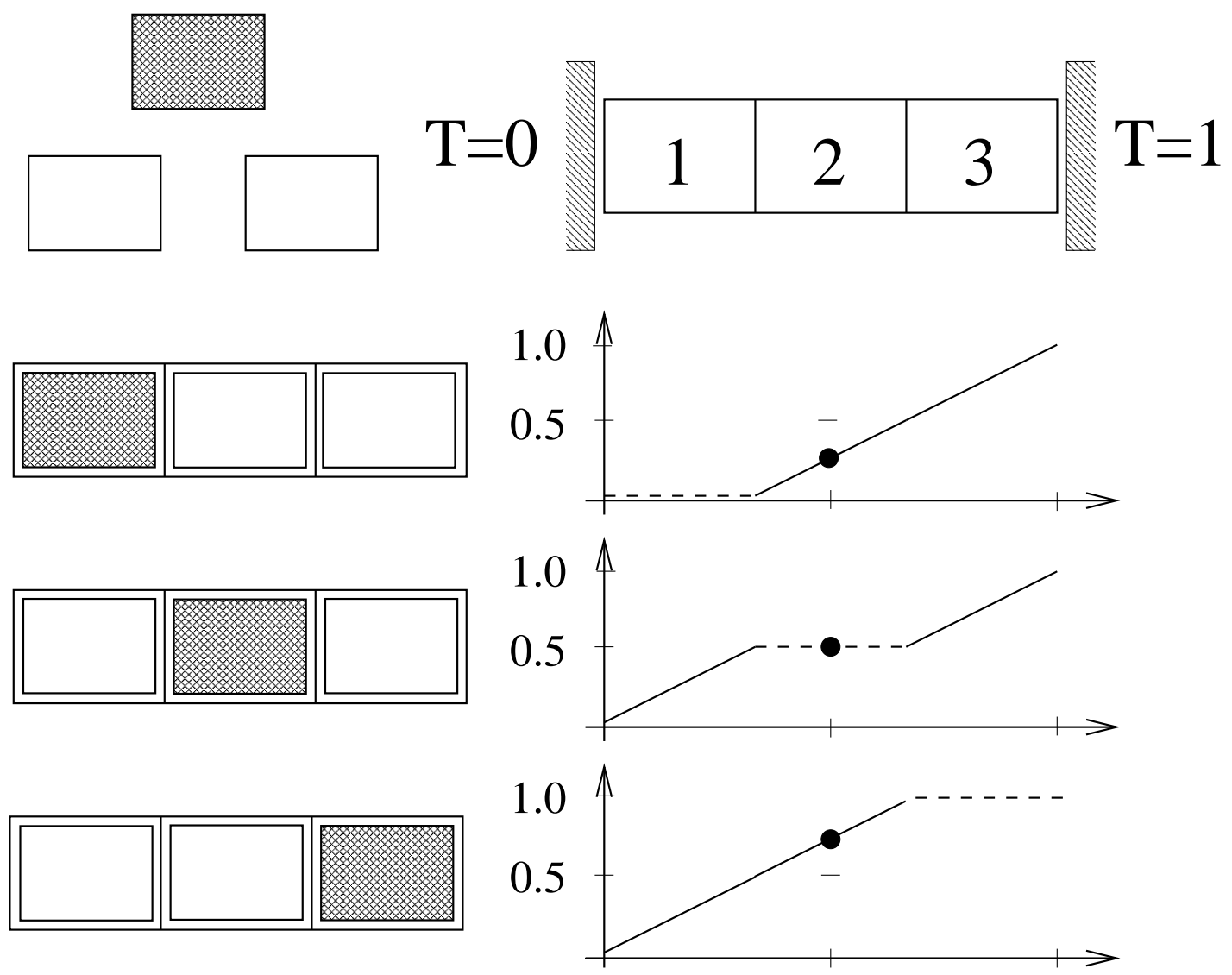

Figure 1: Illustration of the fact that non-uniformity in the spatial distribution of the phases can lead to a difference between the phase-ensemble mean temperatures.

assuming equality of the phase temperatures have yielded questionable results (Buyevich \& Ustinov 1995).

3. Let us now return to the fluid mechanics of a disperse flow. In order to be more definite, it is useful to have a closer look at the closure problem. Consider, for example, the averaged continuousphase (index $C$ ) momentum equation that may be written (Zhang \& Prosperetti 1997; Prosperetti 1998)

$$
\begin{aligned}
\rho_{C}\left[\frac { \partial } { \partial t } \left(\beta_{C}<\right.\right. & \left.\left.\mathbf{u}_{C}>\right)+\boldsymbol{\nabla} \cdot\left(\beta_{C}\left\langle\mathbf{u}_{C} \mathbf{u}_{C}\right\rangle\right)\right] \\
& =\boldsymbol{\nabla} \cdot\left(-\beta_{C}<p_{C}>\mathbf{I}+2 \mu_{C} \mathbf{E}_{m}+\beta_{D} \mathcal{L}\left[\boldsymbol{\sigma}_{C}\right]\right)-\beta_{D} \mathcal{A}\left[\boldsymbol{\sigma}_{C}\right]+\beta_{C} \rho_{C} \mathbf{g} .
\end{aligned}
$$

Here angle brackets denote phase-ensemble averages, $\beta_{C, D}$, with $\beta_{C}=1-\beta_{D}$, are the continuousand disperse-phase volume fractions, and $\mathbf{E}_{m}$ is the rate-of-strain tensor based on the volumetric flow rate of the mixture

$$
\mathbf{u}_{m}=\beta_{C}<\mathbf{u}_{C}>+\beta_{D}<\mathbf{u}_{D}>.
$$

The crux of the closure problem resides in the quantities denoted by capital cursive letters, for example

$$
\beta_{D} \mathcal{A}\left[\boldsymbol{\sigma}_{C}\right](\mathbf{x})=n \overline{\int_{|\mathbf{r}|=a} d S \boldsymbol{\sigma}_{C}(\mathbf{x}+\mathbf{r} \mid N) \cdot \mathbf{n}},
$$

where $n$ is the particle number density, $\boldsymbol{\sigma}_{C}$ is the stress tensor, $\mathbf{n}$ is the outward normal to the particle centered at $\mathbf{x}, N$ denotes a generic configuration of the $N$ particles, and the overline denotes the ensemble average over all the configurations such that one of the particles (all of radius $a$ ) is centered at $\mathbf{x}$. In general, the calculation of such quantities requires a knowledge of the local microscopic 
fields in the neighborhood of each particle, and it is here that reliance on direct numerical simulation proves essential to go beyond the $O\left(\beta_{D}\right)$ dilute-limit validity.

The standard technique to conduct simulations of uniform suspensions is to arrange at random $N$ particles in a cube, and to fill up space with replicas of this cube, which in practice means solving the equations subject to periodicity conditions on the cube surface. Due to the assumed spatial uniformity, ensemble averages equal volume averages, and the latter are readily obtained from the numerical results. This approach evidently will not work in the non-uniform case.

The method we have developed consists in taking the volume averages with a particular weight (i.e., a probability distribution) that turns the initial uniform ensemble into a slightly non-uniform one with a structure particularly suited to the use of Fourier expansions. Since the calculation of Fourier coefficients involves projections - i.e., volume integrals - this method can effectively use the machinery developed for uniform ensembles. The price to pay, however, is that only weak nonuniformities can be considered as the assumed probability distribution is built by subjecting the particles of an initial uniform one to small displacements. Since the perturbed configurations must be realizable, in the sense that the particles cannot compenetrate, the displacement can only be taken to be infinitesimal. This is a limitation but, actually, also a strength as the terms due to the non-uniformity are explicitly identifiable by the fact that they appear multiplied by a small parameter, aside from which they are of order one. If one were to simulate directly non-uniform particle distributions, on the other hand, it would be difficult to isolate the effects of the nonuniformity from statistical noise.

We have used this basic idea in two different ways:

- To identify qualitative phenomenological features of non-uniform suspensions, and

- To derive in a systematic way closure relations.

4. In order to study some basic phenomenological aspects of non-uniform suspensions we have used a computer code based on one kindly supplied to us by Prof. Sangani (Syracuse University). The code (that has been completely rewritten with substantial gains in efficiency) allows the user to conduct direct numerical simulation of Stokes flow past $N$ spheres in a cubic domain with periodic boundary conditions. It can be adapted to situations of imposed plane shear, equal forces applied to the particles (sedimentation), equal torques, equal velocities (flow in a porous medium), and equal angular velocities. So far we have considered the first 3 cases and have found several interesting results:

- As mentioned before, the effective viscosity can be calculated by looking at the response of a uniform suspension to an imposed macroscopic shear. It would be evidently impossible to calculate this quantity by simulating e.g. the sedimentation of a uniform suspension. Bulk viscous stresses are not zero in a non-uniform sedimenting suspension, however, and indeed we have shown how to determine the effective viscosity for such a system as well as for a nonuniform suspension the particles of which are subject to an equal imposed torque. Remarkably, the viscosity values obtained in these three different cases all agree with each other (Fig. 2). This result is of particular importance as it constitutes the first proof that the effective viscosity is a property of a suspension independent of the flow situation considered. Of course, the effective viscosity is also dependent on the suspension microstructure, which we have taken to be that for hard spheres in our simulation. This choice is motivated by the fact that our ultimate objective is to derive equations of the so-called two-fluid form, which is widely used in the energy-related disciplines of engineering. These equations do not contain information on the microstructure, for which some suitably general hypothesis must therefore be introduced. This circumstance may limit the validity of our results to well below the close-packing volume fraction (around 65\%), but important applications (e.g., to fluidized beds) exist already in the range below about $40 \%$. Furthermore, even with this limitation, we are opening the way to the study of non-uniform systems on which, as noted before, little systematic progress has been made. 


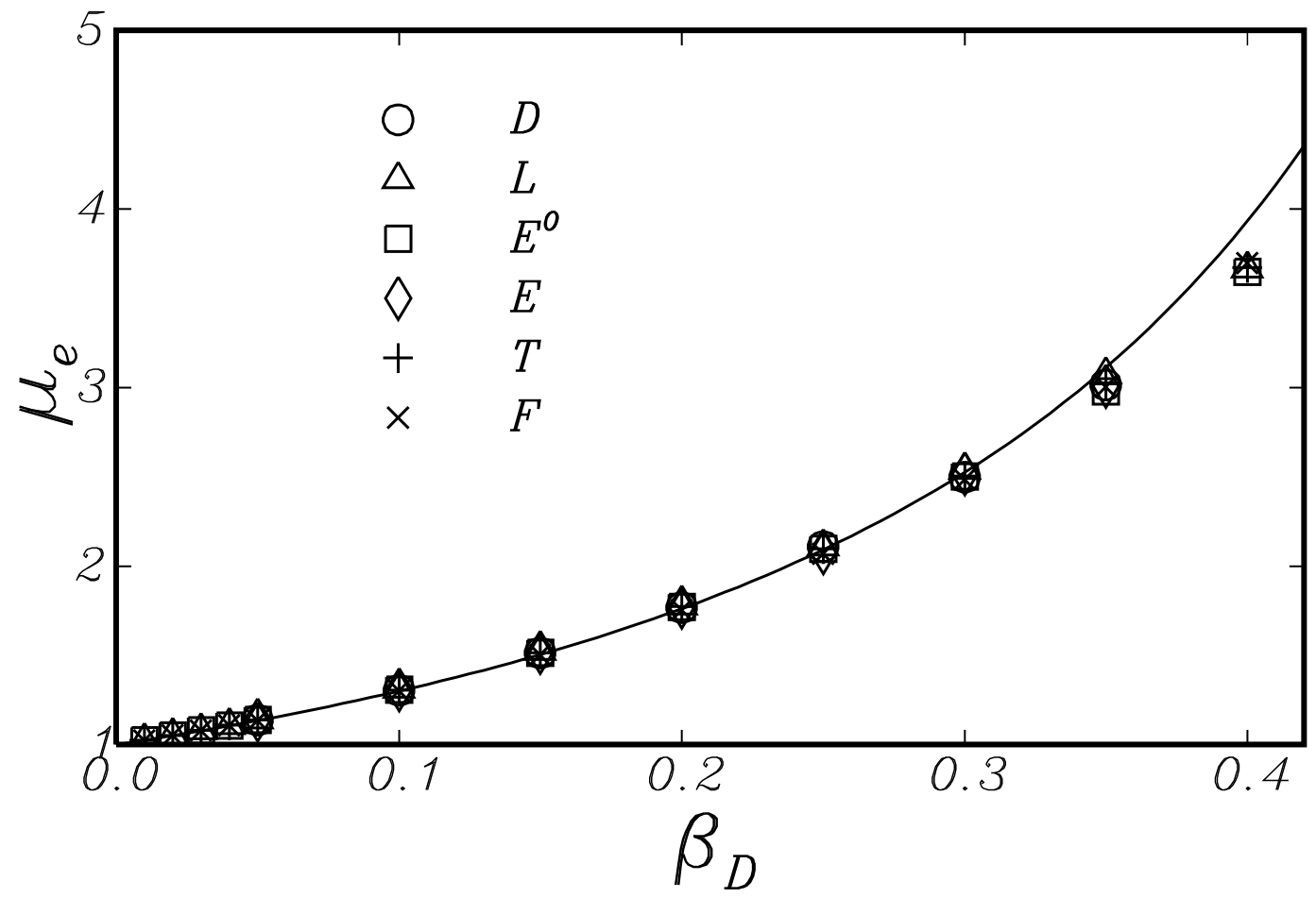

Figure 2: Calculated effective viscosity as a function of the particle volume fraction. The different symbols show values obtained from the simulation of several distinct physical processes: The line is a fit of the form $\mu_{e f f} / \mu_{C}=\left(1-\beta_{D} / 0.79\right)^{-1.9}$.

- While the stress tensor in a uniform suspension can be reduced to a Newtonian form with an effective viscosity, we have proven that this is not possible in the non-uniform case. The modeling of the rheological behavior of a non-uniform suspension necessarily involves additional terms. This fact had been hypothesized before, but - again for the first time - we give a quantitative proof of it independent of particular models or assumptions.

- The average mixture pressure in a suspension does not coincide with the average continuousphase pressure. The properly defined mixture pressure contains also a contribution of the near-field pressure disturbance induced by the particles.

- The settling or shearing of a non-uniform suspension causes a non-zero differential particle rotation with respect to the local mixture angular velocity that contributes to the stress.

It should be explicitly noted that the previous deductions are not based on modeling assumptions, but are purely the consequence of the particular form of averaging that we have developed and of our techniques to simulate non-uniform suspensions. They are therefore solid results that cannot be questioned by future research. We believe that similar techniques can be adapted to the case of finite Reynolds numbers that is, of course, of particular interest for energy-related problems such as fluidized beds.

5. Our ability to simulate the behavior of non-uniform suspensions has enabled us also to develop a systematic approach to the closure problem. In broad outline, the method is the following.

Let a particular flow be characterized by parameters $q_{1}, q_{2}, \ldots, q_{K}$. In practice these quantities could specify the size of the fundamental cubic cell, an applied force, and so forth. All the average quantities that arise in the numerical solution of the problem, such as the mean volumetric flow velocity $\mathbf{u}_{m}$, the mean disperse-phase velocity $\left\langle\mathbf{u}_{D}\right\rangle$, the stress $\mathcal{L}$, etc. will then also be functions of the same parameters:

$$
\begin{aligned}
\mathbf{u}_{m} & =\mathbf{u}_{m}\left(q_{1}, q_{2} \ldots q_{K}\right), \\
<\mathbf{u}_{D}> & =<\mathbf{u}_{D}>\left(q_{1}, q_{2} \ldots q_{K}\right), \\
& \ldots \\
\mathcal{L} & =\mathcal{L}\left(q_{1}, q_{2} \ldots q_{K}\right), \\
\ldots &
\end{aligned}
$$


Choose $K$ quantities as primary (e.g. pressure, velocities, volume fraction, etc.). By eliminating the $q_{j}$ 's between these $K$ primary quantities and the remaining ones, a series of functional relations is obtained that are, at least formally, problem independent:

$$
\mathcal{L}=\mathcal{L}\left(\mathbf{u}_{m},<\mathbf{u}_{D}>, \ldots\right) .
$$

If these relations are indeed intrinsic constitutive relations, the particular problem or parameters used to derive them are of no particular importance and can, therefore, be chosen with convenience in mind.

To illustrate the concept in a very simple case suppose that, in a molecular dynamics simulation, one were to set up a temperature distribution

$$
T(\mathbf{x})=T_{0}+A \sin \mathbf{k} \cdot \mathbf{x},
$$

with $\mathbf{k}, A$ given and, by conducting the simulation, found that the heat flux has the form

$$
\mathbf{q}=-B \mathbf{k} \cos \mathbf{k} \cdot \mathbf{x}
$$

with $B$ calculated from the simulation. This results - conceptually analogous to (.4) - would represent only the solution to a particular problem, namely that in which $T(\mathbf{x})$ is given by (.6). Its significance can, however, be greatly increased by observing that, by eliminating parameters between (.6) and (.7) one would find a result conceptually similar to (.5), i.e.

$$
\mathbf{q}=-\frac{B}{A} \boldsymbol{\nabla} T
$$

which has the form of a constitutive relation of broader applicability than (.7) and determines the thermal conductivity since $B$ has been found numerically. It is by using this general idea that we have calculated, for non-dilute suspensions, the generalization of the Faxén term, the "volume viscosity", the effect of interphase slip on the mixture stress, and other properties, as briefly noted below.

In spite of its simplicity, the previous example shows a potential difficulty that, indeed, we have found in our work, namely a possible dependence of the coefficients appearing in the constitutive relation upon the dimensionless scale $a|\mathbf{k}|$, where $a$ could be, e.g., the mean free path in a molecular dynamics simulation, or the particle radius in a suspension, and so forth. For practical applications one would be interested in the macroscopic limit of $B / A$ as $a|\mathbf{k}| \rightarrow 0$, which requires running several simulations and extrapolating. Attaining sufficiently small values of $a|\mathbf{k}|$ requires the ability to simulate many particles and imposes a heavy computational burden on the method. In spite of this difficulty, we have been able to take the limit with confidence for some quantities, for which we have found firm results. Other quantities exhibit a stronger dependence on $a|\mathbf{k}|$ and the simulation of larger sphere assemblies is needed.

6. Another point that can be made with the previous simple example is that, in order to find information useful for the closure of the equations, one does not need to choose the flow to be simulated for practical relevance, but rather with an eye toward its usefulness for the development of constitutive relations. This remark is of crucial importance in the present situation in which computers and numerical techniques are not sufficiently developed to carry out direct simulations of general realistic flows. We believe that, by studying simple flows - which are very definitely within the range of the possible - one can develop closure relations or, at the very least, one can perfect closure methods that will permit good closures in a later stage of greater development of computational capabilities.

7. For the three cases of non-uniform suspensions subject to shear, and applied forces or torques on the particles, by means of the parameter elimination concept described before, we have developed closure relations conceptually similar to (.5). For example, we have shown that the total stress $-\beta_{C}<p_{C}>\mathbf{I}+2 \mu_{C} \mathbf{E}_{m}+\beta_{D} \mathcal{L}\left[\boldsymbol{\sigma}_{C}\right]$ (see Eq. .1) can be decomposed as

$$
-\beta_{C}<p_{C}>\mathbf{I}+2 \mu_{C} \mathbf{E}_{m}+\beta_{D} \mathcal{L}\left[\boldsymbol{\sigma}_{C}\right]=-p_{m} \mathbf{I}+q_{m} \mathbf{I}+2 \mu_{e f f} \mathbf{E}_{m}+\mathbf{S}+\mathbf{A},
$$


where $p_{m}$ is the mixture pressure, $q_{m}$ another isotropic part of the stress (akin to the term giving rise to the volume viscosity in a gas), $\mu_{\text {eff }}$ the effective viscosity, and $\mathbf{S}, \mathbf{A}$ additional symmetric and antisymmetric contributions. For the term $q_{m}$ we have found

$$
q_{m}=\mu_{I}\left(\beta_{D}\right) \nabla \cdot \mathbf{u}_{\Delta},
$$

where $\mu_{I}$ is determined numerically and $\mathbf{u}_{\Delta}=\left\langle\mathbf{u}_{D}\right\rangle-\mathbf{u}_{m}$ is the interphase slip velocity. This relation was arrived at by exploiting several a priori constraints such as linearity, Galilean invariance, scalar (as opposed to pseudo-scalar) nature of the result, and so forth. For the term $\mathbf{S}$ we have, so far, the partial result

$$
\mathbf{S}=2 \mu_{\Delta}\left(\beta_{D}\right) \mathbf{E}_{\Delta}+\ldots
$$

where $\mu_{\Delta}\left(\beta_{D}\right)$ is calculated numerically and $\mathbf{E}_{\Delta}$ is the rate-of-strain tensor based on the inter-phase slip velocity $\mathbf{u}_{\Delta}$. The presence of excessive numerical noise has prevented us until now from getting reliable closure relations for the remainder of $\mathbf{S}$ and $\mathbf{A}$. We need to refine our statistics and to model other physical situations (e.g., porous medium or an imposed angular velocity) to get a better handle on these terms.

Similarly, for the inter-phase force $\mathbf{f}$ we have found

$$
\mathbf{f}=-\frac{6 \pi \mu_{C} a \beta_{D} v}{\Phi\left(\beta_{D}\right)} \mathbf{u}_{\Delta}+2 \boldsymbol{\nabla} \cdot\left[Q\left(\beta_{D}\right) \nabla \mathbf{E}_{m}\right]+\ldots
$$

Here $\Phi$ is the hindered settling function, for which our results coincide with those of other investigators, and the following term is the generalization to finite volume fractions of the familiar Faxén term. Note that, in expanded form, this term is

$$
\boldsymbol{\nabla} \cdot\left[Q\left(\beta_{D}\right) \boldsymbol{\nabla} \mathbf{E}_{m}\right]=Q \nabla^{2} \mathbf{u}_{m}+Q^{\prime} \mathbf{E}_{m} \cdot \boldsymbol{\nabla} \beta_{D},
$$

and neither term could be found from the simulation of a uniform suspension. Note also that the relation between the two coefficients (i.e., that the second one is derivative of the first one with respect to $\beta_{D}$ ) was proven on the basis of the numerical results and not imposed a priori. The dots in Eq. (.12) are other terms that, once again, so far we only know imperfectly due to numerical difficulties.

\section{Publications Resulting from This Grant}

This grant resulted in a number of publications by the PI and his co-workers and students.

\section{Journal papers}

1. Sangani, A, Zhang, D.Z., and Prosperetti, A. The added mass, Basset, and viscous drag coefficients in nondilute bubbly liquids undergoing small-amplitude oscillatory motion, Phys. Fluids, A3, 2955-2970, 1991

2. Kim, H.S. and Prosperetti, A. Numerical simulation of the motion of rigid spheres in potential flow, SIAM J. Appl. Math. 52, 1533-1562, 1992

3. Watanabe, M. and Prosperetti, A. Shock waves in dilute bubbly liquids, J. Fluid Mech. 274, 349-381, 1994

4. Zhang, D.Z. and Prosperetti, A. Averaged equations for inviscid disperse two-phase flow, $J$. Fluid Mech. 267, 185-219, 1994 (with an appendix by H. Bulthuis)

5. Huang, H.X. and Prosperetti, A. Effect of orthogonality and smoothness of computational grids on the numerical solution of the convection-diffusion equation, J. Numer. Heat Transfer B26, 1-20, 1994 
6. Yuan, H. and Prosperetti, A. On the in-line motion of two spherical bubbles in a viscous liquid, J. Fluid Mech. 278, 325-349, 1994

7. Takagi, S., Prosperetti, A. and Matsumoto, Y. Drag coefficient of a gas bubble in an axisymmetric shear flow, Phys. Fluids 6, 3186-3188, 1994

8. Bulthuis, H.F., Prosperetti, A. and Sangani, A.S. "Particle stress" in disperse two-phase potential flow, J. Fluid Mech. 294, 1-16, 1995

9. Zhang, D.Z. and Prosperetti, A. Ensemble phase averaged equations for bubbly flows, Phys. Fluids 6, 2956-2970, 1994

10. Sarkar, K. and Prosperetti, A. Effective boundary conditions for the Laplace equation with a rough boundary, Proc. Roy. Soc. 451, 425-452, 1995

11. Duraiswami, R. and Prosperetti, A. Linear Pressure Waves in Fogs, J. Fluid Mech. 299, $187-215,1995$

12. Prosperetti, A. and Zhang, D.Z. Finite-Particle-Size Effects in Disperse Two-Phase Flows, Theoret. Comput. Fluid Dynamics 7, 429-440, 1995

13. K. Sarkar \& A. Prosperetti, Effective boundary conditions for the Stokes flow over a rough surface, J. Fluid Mech. 316, 223-240, 1996

14. D.Z. Zhang \& A. Prosperetti, Disperse-phase stress in two-phase flow, Chem. Eng. Comm. 141-142, 387-398, 1996

15. D.Z. Zhang \& A. Prosperetti, Momentum and energy equations for disperse two-phase flows and their closure for dilute suspensions, Int. J. Multiphase Flow 23, 425-253, 1997

16. M. Marchioro \& A. Prosperetti, Heat conduction in a non-uniform composite with spherical inclusions, Proc. Royal Soc. London, A455, 1483-1508, 1999

17. M. Marchioro, M. Tanksley, \& A. Prosperetti, Mixture pressure and stress in spatially nonuniform suspensions, Int. J. Multiphase Flow 25, 1395-1429, 1999.

18. M. Marchioro, M. Tanksley, \& A. Prosperetti, Flow of spatially non-uniform suspensions. Part I: Phenomenology, Int. J. Multiphase Flow 26, 783-831, 2000.

19. M. Marchioro, M. Tanksley, \& A. Prosperetti, Flow of spatially non-uniform suspensions. Part II: An approach to the closure of the average relations, Int. J. Multiphase Flow 27, 237-276, 2001.

20. D.K. Han \& A. Prosperetti, Shape decomposition technique in electrical impedance tomography, J. Comput. Phys. 155, 75-95, 1999.

\section{Papers in volumes}

1. Prosperetti, A. and Zhang, D.Z., Phase averaging and average pressure gradient in disperse two-phase flow, in Proceedings of the NSF-DOE Workshop on Flow of Particulates and Fluids, Gaithersburg, 1992

2. Prosperetti, A., Bubble mechanics: luminescence, noise, and two-phase flow, in Theoretical and Applied Mechanics, Bodner, S.R., Singer, J., Solan, A. and Hashin, Z. eds., North-Holland (Amsterdam) 1993, pp. 355-369

3. Sangani, A.S. and Prosperetti, A., Numerical simulation of the motion of particles at large Reynolds numbers, in Particulate Two-Phase Flow, Roco, M. ed., Butterworth-Heinemann (Boston) 1993, pp. 971-998 
4. Zhang, D.Z. and Prosperetti, A., Ensemble averaged Euler equations and added mass for linearized flows, in Proceedings of the ASME Summer Meeting, Washington, 1993

5. A. Prosperetti, Multiphase flows, cavitation, and bubbles, in Research Trends in Fluid Dynamics, J.L. Lumley, A. Acrivos, L.G. Leal \& S. Leibovich eds., American Institute of Physics, pp. 244-252, 1996

6. A. Prosperetti, Ensemble averaging techniques for disperse flows, in Particulate Flows: Processing and Rheology, edited by D.A. Drew, D.D. Joseph \& S.L. Passman, Springer, pp. 99-135, 1997

7. Prosperetti, A. Ensemble Averaging Techniques for Disperse Flows, in Particulate Flows: Processing and Rheology, Drew, D.A., Joseph, D.D., and Passman, S.L. Eds., Springer, New York, pp. 99-136, 1998.

A number of other papers have been presented at conferences (JSME, APS, and others), but only abstracts are available for them and, therefore, they are not listed here.

\section{Personnel}

In addition to the PI, the work reported in the papers listed above has involved the following persons:

- Senior collaborators: A.S. Sangani, Y.Matsumoto

- Post-doctoral fellows: H.S. Kim, H.X. Huang, D.Z. Zhang, M. Tanksley

- Graduate students: M. Watanabe, R. Duraiswami, D.Z. Zhang, H. Yuan, S. Takagi, K. Sarkar, D.K. Han, M. Marchioro, W. Wang

- Ph. D. Degrees Awarded: M. Watanabe, R. Duraiswami, D.Z. Zhang, H. Yuan, K. Sarkar, D.K. Han, M. Marchioro

- M.S. Degrees Awarded: H. Bulthuis, W. Wang

\section{Patent Report}

This work did not result in any invention or patent.

\section{Equipment Inventory}

No government-furnished property was used for the performance of this work. 


\section{References}

Batchelor, G.K., 1970, The stress system in a suspension of force-free particles, J. Fluid Mech., 41, 545-570.

Batchelor, G.K., 1988, A new theory of the instability of a uniform fluidized bed, J. Fluid Mech., 193, 75-110.

Bulthuis, H.F., Prosperetti, A., \& Sangani, A.S., 1995, "particle stress" in disperse two-phase potential flow, J. Fluid Mech., 294, 1-16.

Buyevich, Yu. A. \& Ustinov, V.A., 1995, Effective thermal conductivity of a microscopically inhomogeneous dispersion, Int. J. Heat Mass Transfer, 38, 381-389.

Jones, A.V. \& Prosperetti, A., 1985, On the suitability of first-order differential models for two-phase flow prediction, Int. J. Multiphase Flow, 11, 133-148.

Mo, G. \& Sangani, A.S., 1994, A method for computing Stokes flow interactions among spherical objects and its application to suspensions of drops and porous particles, Phys. Fluids, 6, $1637-1652$.

Prosperetti, A., Ensemble averaging techniques for disperse flows, in Particulate Flows: Processing and Rheology, Drew, D., Joseph, D.D., \& Passman, S.L., eds., Springer, 1998, 99-136.

Prosperetti, A. \& Jones, A.V., 1987, The linear stability of general two-phase flow models II, Int. J. Multiphase Flow, 13, 161-171.

Prosperetti, A. \& Satrape, J.V., Stability of two-phase flow models, in Two Phase Flows and Waves, Joseph, D.D. \& Schaeffer, D.G., eds., Springer, 1990, 98-117.

Sangani, A.S. \& Didwania, A.K., 1993, Dispersed-phase stress tensor in flows of bubbly liquids at large Reynolds numbers, J. Fluid Mech., 248, 27-54.

Zhang, D.Z. \& Prosperetti, A., 1997, Momentum and energy equations for disperse two-phase flows and their closure for dilute suspensions, Int. J. Multiphase Flow, 23, 425-453. 